\title{
Current trends in the treatment of hepatic encephalopathy
}

This article was published in the following Dove Press journal:

Therapeutics and Clinical Risk Management

30 July 2009

Number of times this article has been viewed

\section{Mohamad Rasm Al Sibae \\ Brendan M McGuire \\ Department of Medicine, Division of Gastroenterology and Hepatology, University of Alabama at Birmingham, Birmingham, AL, USA}

Abstract: Hepatic encephalopathy (HE) is a common reversible neuropsychiatric syndrome associated with chronic and acute liver dysfunction and significant morbidity and mortality. Although a clear pathogenesis is yet to be determined, elevated ammonia in the serum and central nervous system are the mainstay for pathogenesis and treatment. Management includes early diagnosis and prompt treatment of precipitating factors (infection, gastrointestinal bleeding, electrolyte disturbances, hepatocellular carcinoma, dehydration, hypotension, and use of benzodiazepines, psychoactive drugs, and/or alcohol). Clinical trials have established the efficacy of lactulose and lactitol enemas in the treatment of acute hepatic encephalopathy. Extensive clinical experience has demonstrated the efficacy of oral lactulose and lactitol with the goal of two to three soft bowel movements a day for the treatment of chronic HE. However, lactulose and lactitol have significant gastrointestinal side effects. For patients unable to tolerate lactulose or lactitol or who still have persistent chronic HE with lactulose or lactitol, neomycin, metronidazole and rifaximin are second-line agents. More recent data supports the benefits of rifaximin used solely and as an additional agent with fewer side effects than neomycin or metronidazole. Newer therapies being investigated in humans with clinical promise include nitazoxanide, the molecular adsorbent recirculating system (MARS), L-ornithine phenylacetate, sodium benzoate, and/or sodium phenylacetate and Kremezin ${ }^{\circledR}$ (AST-120).

Keywords: hepatic encephalopathy, liver dysfunction, lactulose, lactitol

\section{Introduction}

Hepatic encephalopathy (HE) is a reversible neuropsychiatric syndrome associated with chronic and acute liver dysfunction. It is characterized by cognitive and motor deficits of varying severity. Early symptoms include reversal of sleep pattern, apathy, hypersomnia, irritability, and personal neglect. In later stages, delirium and coma can arise with neurologic signs including hyperreflexia, rigidity, myoclonus, and asterixis. ${ }^{1}$ An important aspect of this syndrome is the diversion of portal blood into systemic circulation through portosystemic collateral vessels, without being filtered by hepatocytes. HE has also been seen clinically among noncirrhotic patients with either spontaneous or surgically created portosystemic shunts. ${ }^{2}$

Subtle signs of HE are seen in almost $70 \%$ of patients with cirrhosis and can have a significant impact on a patient's functional ability. More severe symptoms can be incapacitating in a considerable number of patients with end-stage liver disease and are noticed in nearly $30 \%$ of patients who experience portosystemic shunt surgery. ${ }^{3}$ Roughly $30 \%$ of patients with end-stage liver disease experience significant encephalopathy approaching coma that require hospitalization. ${ }^{4}$ Therefore, HE with cirrhosis is considered a poor
Correspondence: Brendan M McGuire University of Alabama at Birmingham Department of Medicine, Division of Gastroenterology and Hepatology, I530 Third Avenue South, MCLM 262A, Birmingham, AL 35294-0005, USA Email bmcguire@uab.edu 
prognostic sign and without liver transplantation the one year survival rate is $42 \%$ after the first episode of overt $\mathrm{HE}$ and the three-year survival rate is $23 \% .^{5}$

There are two classification systems that are frequently used to assess hepatic encephalopathy. The World Congress of Gastroenterology has categorized HE based on underlying hepatic abnormalities and for patients with cirrhosis it can be further subdivided by the duration and characteristics of neurologic dysfunction. The three types of $\mathrm{HE}$ are $\mathbf{A}, \mathbf{B}$, and $\mathbf{C}$ which are associated with Acute liver failure, portal systemic Bypass without intrinsic liver disease, and Cirrhosis, respectively. ${ }^{6-8}$

Encephalopathy has been further subdivided, based on duration and characteristics of neurologic dysfunction, into episodic, persistent, and minimal subtypes. Episodic HE occurs over a short time span and fluctuates in severity. Persistent HE is a chronic clinical condition of cognitive deficits that affects social and occupational functioning. Minimal HE is associated by subtle cognitive impairments of attention, response inhibition and executive function. The other frequently used classification system is the West Haven criteria. The West Haven criteria is a routinely used grading system to assess the severity of symptoms in patients with overt HE (Table 1). ${ }^{9}$ The majority of these symptoms are manageable with treatment, and reversible with liver transplantation.

\section{Pathophysiology}

Decades of clinical experience and research has failed to establish a clear pathogenesis of HE. The pathophysiology

Table I West Haven criteria for altered mental status in hepatic encephalopathy

Minimal hepatic encephalopathy

Minimal changes in memory and lack of detectable changes in personality or behavior. No asterixis.

Grade I

Trivial lack of awareness, shortened attention span, sleep disturbance, altered mood, and slowing the ability to perform mental tasks. Asterixis can be detected.

\section{Grade 2}

Lethargy or apathy, disorientation to time, amnesia of recent events, impaired simple computations, inappropriate behavior, slurred speech. Asterixis is present.

\section{Grade 3}

Somnolence, confusion, disorientation to place, bizarre behavior, clonus, nystagmus, and positive Babinski sign. Asterixis usually absent.

\section{Grade 4}

Coma with or without response to painful stimuli. is not a single clinical entity, rather a complicated interplay of many factors and the abnormalities involving ammonia metabolism is the most frequently implicated factor. The normal gut flora produces a urease that enzymatically cleaves ammonia $\left(\mathrm{NH}_{3}\right)$ from protein in the small bowel and colonic lumen. Ammonia is then absorbed into the portal circulation. It reaches the systemic circulation via portosystemic shunting and the inability of the liver to metabolize ammonia. ${ }^{10}$ Excessive plasma ammonia can accumulate in the central nervous system (CNS) causing significant alteration in neurotransmission. Excessive levels of ammonia have can be seen in the basal ganglia and cerebellum of cirrhotic patients with HE. ${ }^{11,12}$ It is believed that this contributes to the increase prevalence of motor dysfunction and the extra pyramidal symptoms exhibited with HE.

Other clinical observations possibly involved in the pathogenesis of HE are decreased serum levels of branchedchain amino acids and increased brain levels of aromatic amino acids. ${ }^{13}$ The principal neuro-inhibitory neurotransmitter gamma-aminobutyric acid (GABA) is also increased in the cerebral spinal fluid (CSF) of patients with encephalopathy. Other toxins identified in the CNS include increased levels of endogenous benzodiazepine-like compounds, manganese, oxygen free radicals, circulation opioid peptides, nitric oxide, inflammatory cytokines, reduction in serotoninergic neurotransmitters, and depletion of endogenous antioxidants. ${ }^{14-17}$ Thus, the pathophysiology of HE is a result of several complicated factors rather than a single mechanism.

\section{Diagnosis}

The diagnosis of minimal HE can only be made by administering psychometric tests, including the portosystemic HE score (PHES). The testing of patients is time consuming and requires psychological expertise for administering and interpreting the results. The critical flicker frequency is a highly objective and sensitive measure of minimal hepatic encephalopathy, but requires purchase of equipment and a closed room without distractions. ${ }^{18-20}$ Magnetic resonance spectroscopy (MRS) is currently being studied, but due to variability among initial studies and costs it not used clinically. ${ }^{21}$ Overt HE is a diagnosis of exclusion. Similar neuropsychiatric symptoms are seen in a variety of metabolic disorders, toxic ingestions, infectious processes or intracranial processes. In patients with cirrhosis and portosystemic shunting, a known precipitating factor and a typical clinical presentation is usually sufficient to make a diagnosis. Lab testing is necessary to exclude treatable causes 
such as hypoxia, azotemia, hyponatremia, hypoglycemia, and other psychoactive drugs. Arterial or venous ammonia levels may be helpful in securing the diagnosis and correlate with the severity of the disease. ${ }^{22}$ A physical exam is important to identify other potential causes. Further work up may be warranted to exclude other potential causes in difficult to diagnose patients, including additional laboratory studies, electroencephalogram, lumbar puncture, and/or imaging of the head.

\section{Treatment}

The protocols used to treat patients with HE were derived in the 1970s, but many of the studies had flaws in their design. ${ }^{23,24}$ Most of the studies lacked standard definitions and grading scales of hepatic encephalopathy, included small sample sizes, and rarely used a placebo control group. Despite these limitations the studies findings that removal of ammonia from the body is critical to the treatment of HE is still widely adhered to by clinicians. Hyperammonemia is detected in over $80 \%$ of patients with HE. Therefore, treatment is aimed at:

- Excluding nonhepatic causes of altered mental function.

- Treating precipitating factors.

- Initiating treatments based on the ammonia hypothesis.

- Managing severe encephalopathy in the intensive care unit with full medical support.

\section{Treatment of precipitating causes}

The most important aspect of HE management is prompt recognition and treatment of precipitating factors rather than assuming a deterioration of hepatocellular function. Fessel and colleagues demonstrated that $\mathrm{HE}$ is caused by reversible factors in over $80 \%$ of patients. ${ }^{25}$ These common reversible factors include constipation, infection, hypokalemia, gastrointestinal bleeding, increased protein intake, sedatives, and tranquilizers. Thus, identifying and correcting the reversible precipitating factors can be beneficial in treating most episodes of HE.

\section{Treatment based on the ammonia hypothesis Dietary issues}

There is no substantial evidence to support protein restriction among cirrhotic patients. Therefore a diet with approximately 1 to $1.5 \mathrm{~g}$ of dietary protein per kilogram of body weight per day can be administrated safely to a patient with hepatic encephalopathy. In a randomized study, Cordoba and colleagues demonstrated that a protein-restricted diet does not improve $\mathrm{HE}$ in cirrhotic patients. ${ }^{26}$ The source of proteins may be important to controlling HE. Bianchi presented in a randomized crossover trial that vegetable proteins were superior to animal-derived proteins. ${ }^{27}$ In addition, a high fiber diet may be beneficial by increasing the transit rate of food through the intestines and lowering the $\mathrm{pH}$ of the colonic lumen as a result of its fermentation by colonic bacteria. ${ }^{28}$ Branched-chain amino acids appear to be more tolerable with a positive nitrogen balance however supplementation with branched-chain amino acids have failed to show any improvement in morbidity or mortality among patients with HE. ${ }^{29,30}$ As a general rule, patients who develop deteriorating HE from an animal-derived protein diet warrant substituting a well-balanced diet with vegetable proteins and a high fiber diet.

\section{Pharmacologic approach}

Nonabsorbable disaccharides in the reduction of intestinal ammonia production and absorption

Lactulose (beta-galactosidofructose) and lactitol (beta-galactosidosorbitol) are nonabsorbable disaccharides that are currently used as first line agents for the treatment of HE. Lactitol is not available in the United States. The exact mode of action by lactulose is thought to be the conversion to lactic acid and acetic acid resulting in acidification of the gut lumen. This favors conversion of ammonia $\left(\mathrm{NH}_{3}\right)$ to ammonium $\left(\mathrm{NH}_{4}^{+}\right)$, which is relatively membrane impermeable; therefore, less ammonia is absorbed by the colon. Gut acidification inhibits ammoniagenic coliform bacteria, leading to increased levels of nonammoniagenic lactobacilli. Nonabsorbable disaccharides also work as a cathartic, clearing the gut of ammonia before it can be absorbed. Als-Nielsen and colleagues were able to demonstrate, in a systemic review, that lactulose and lactitol are more effective than a placebo in treating HE. ${ }^{31}$ However, they failed to show any benefit in survival rates. In a controlled trial of 61 patients, Prasad and colleagues showed lactulose improves cognitive function and health related quality of life among patients with cirrhosis who have minimal HE. ${ }^{32}$ In a double-blinded randomized trial, Morgan and colleagues showed lactitol was more tolerable and produced fewer side effects than lactulose. ${ }^{33}$ In a double-blinded randomized study, Uribe and colleagues showed lactulose and lactitol enemas were more effective than tap water in 20 patients. This suggests that colonic acidification is more important than bowel cleansing. ${ }^{34}$

Treatment of acute episodes of HE include nonabsorbable disaccharides administered orally or through a nasogastric 
tube at an initial dose of $45 \mathrm{ml}$, followed by a repeated dose every hour until the patient has a bowel movement. For patients at risk of aspiration, lactulose (or lactitol) can be administered as an enema (300 $\mathrm{ml}$ in $700 \mathrm{ml}$ of water, retained for one hour in the Trendelenburg position) every two hours as needed until mental function improves. After clinical improvement of $\mathrm{HE}$ and once the risk of aspiration becomes minimal, lactulose (or lactitol) can be administered orally and titrated to achieve two to three soft bowel movements daily (the usual oral dosage is $15-45 \mathrm{ml}$ every 8-12 hours). For patients with chronic encephalopathy and for prevention of episodic HE the daily dosage of nonabsorbable disaccharides should be titrated to maintain 2-4 soft bowel movements daily. The main side effects of nonabsorbable disaccharides include an excessively sweet taste, flatulence, abdominal cramping, and electrolyte imbalance, particularly hypernatremia, which can deteriorate the patient's mental status. For patients who can not tolerate the sweet taste of lactulose a powder packet is available.

\section{Antibiotics in the reduction of intestinal ammonia production}

Certain oral antibiotics can reduce urease-producing bacteria in the intestines, resulting in a decrease of ammonia production and absorption through the gastrointestinal tract. Antibiotics are used generally in patients who do not tolerate nonabsorbable disaccharides or in patients who are symptomatic on nonabsorbable disaccharides and require a second agent.

\section{Rifaximin}

Rifaximin is an oral nonsystemic antibiotic with less than $0.4 \%$ absorption. It was granted an orphan drug designation by the US Food and Drug Administration (FDA) for use in hepatic encephalopathy. A phase III multicenter, randomized, double-blinded, placebo-controlled trial involving 299 patients using rifaximin $550 \mathrm{mg}$ orally twice a day or placebo for six months was recently completed and showed that patients who received rifaximin for six months had highly statistically significant protection against clinical HE breakthrough episodes (58\% risk reduction, $\mathrm{p}<0.0001$ ) compared to placebo. ${ }^{35}$ Rifaximin is currently being reviewed by the FDA for approval. In a randomized double-blind, double dummy and controlled trial, Mas and colleagues showed rifaximin is as effective as lactitol in patients with moderate to severe HE. ${ }^{36}$ A review by Lawrence and colleagues concluded that rifaximin is equally effective and in some studies superior to nonabsorbable disaccharides and other antimicrobials in relieving the signs and symptoms observed in cirrhotic patients with mild-to-moderately severe HE. ${ }^{37}$ Additional well-designed studies will be needed to fully delineate the efficacy of rifaximin with or without nonabsorbable disaccharides in patients with HE.

\section{Neomycin}

Neomycin is FDA-approved for the treatment of acute HE, but not chronic HE. Neomycin has been used clinically for many years to treat hepatic encephalopathy, yet there are no controlled studies finding it to be as effective as lactulose. Conn and colleagues demonstrated that neomycin is as effective as lactulose in 33 patients. ${ }^{38}$ However, in a more recent randomized study by Strauss, neomycin was shown to be no better than a placebo in 39 patients with HE. ${ }^{39}$ In acute encephalopathy, neomycin can be administered orally at a dose of $1000 \mathrm{mg}$ orally every six hours for up to six days. For chronic encephalopathy the dosage routinely used is $1-2 \mathrm{~g}$ daily. Although neomycin is poorly absorbed, its chronic administration may result in nephrotoxicity and ototoxicity. ${ }^{40,41}$ Patients who are maintained on chronic neomycin must have periodic evaluation for potential side effects.

\section{Metronidazole}

Metronidazole is not an FDA-approved antibiotic for the treatment of HE. In a report of 11 mild to moderate HE patients and seven chronically affected HE cirrhotic patients treated with metronidazole for one week, Morgan and colleagues showed metronidazole to be as effective as neomycin. ${ }^{42}$ The recommended oral dose of metronidazole for chronic use is $250 \mathrm{mg}$ bid. Prolonged administration of metronidazole can be associated with gastrointestinal disturbance and neurotoxicity.

\section{Nitazoxanide}

Nitazoxanide is an oral agent indicated for the treatment of infectious diarrhea caused by Crytpsporidium parvum and Giardia lamblia. Basu and colleagues presented a pilot prospective study at the 2008 American Association for the Study of Liver Diseases meeting showing clinical improvement in HE among cirrhotic patients who received nitazoxanide and lactulose. ${ }^{43}$ Results such as these are encouraging larger studies.

\section{Combination of disaccharides and antibiotics}

A combination of disaccharides and antibiotics is generally used in difficult to treat patients with $\mathrm{HE}$, but clinical data is limited. In a retrospective chart review of 213 cases, Mantry and colleagues showed that the number of hospitalizations 
and the duration of hospital stays were shortened for patients receiving combination therapy compared with those receiving lactulose monotherapy. Additional studies to further investigate the potential therapeutic benefit of combination therapy will be needed. ${ }^{44,45}$

The phase III multicenter, randomized, double-blinded, placebo controlled trial involving 299 patients using refaximin versus placebo mentioned earlier allowed patients to continue on lactulose. Over $90 \%$ of the study participants received lactulose. A sub-analysis of these patients also showed clinical prevention against HE compared to placebo. ${ }^{35}$

\section{Drugs and devices aimed at elimination of ammonia from plasma \\ Ornithine-aspartate}

Ornithine-aspartate is a stable salt of two amino acids used for the treatment of HE, which is not yet available in the United States. Ornithine-aspartate is both involved in hepatic and muscle metabolism of ammonia, through urea cycle and glutamine synthesis. Ornithine-aspartate is available in both enteral and parenteral forms and has been used to enhance metabolic conversion of ammonia through both pathways. In a trial of 126 patients with cirrhosis and mild chronic hepatic encephalopathy, a daily dose of ornithineaspartate at $20 \mathrm{~g}$ administered intravenously of over a period of one week resulted in lower serum ammonia levels and reduced $\mathrm{HE}$ versus placebo. ${ }^{46}$ In a smaller trial, ornithineaspartate administered at a dose of $18 \mathrm{~g}$ daily for a period of two weeks resulted in a significant decrease in serum fasting and postprandial ammonia levels as well as significant improvement in mental status and cognitive function in patients with chronic overt and minimal encephalopathy when compared to a placebo. ${ }^{47}$ Except for mild gastrointestinal disturbance, no major side effects of this therapy have been reported.

\section{L-ornithine phenylacetate}

L-ornithine phenylacetate has also been considered in the treatment of HE. L-ornithine would participate in the clearance of ammonia in muscles and the liver through glutamine synthesis and the phenylacetate would conjugate with glutamine (which contain nitrogen from ammonia) to form phenylacetylglutamine. Phenylacetylglutamine is excreted by the kidneys by glomerular filtration and tubular secretion. The theoretical benefit of this combination over the ornithine-aspartate combination is the ability to excrete phenylacetylglutamine into the urine. ${ }^{48}$ Human clinical trials are needed to confirm this theory.

\section{Sodium benzoate and/or sodium phenylacetate}

Sodium benzoate and/or sodium phenylacetate are shown to enhance tissue metabolism of ammonia. Benzoate conjugates with glycine (which contain nitrogen from ammonia) to form hippuric acid and phenylacetate conjugates with glutamine (which contain nitrogen from ammonia) to form phenylacetylglutamine. Both hippuric acid and phenylacetylglutamine are excreted by the kidneys by glomerular filtration and tubular secretion. The combination of sodium benzoate with sodium phenylacetate orally was used in a small study, which suggested it may be beneficial in the treatment of $\mathrm{HE} .{ }^{49}$ An oral dose of $5 \mathrm{~g}$ bid of sodium benzoate was as effective as lactulose in reducing serum ammonia levels and improving cognitive function. ${ }^{50}$ Routine use of the oral forms of both or either agent alone has been limited by concern of the sodium load and increased frequency of adverse gastrointestinal symptoms particularly nausea. Ammonul (10\% sodium benzoate and $10 \%$ sodium phenylacetate) is FDA-approved intravenously for the treatment of hyperammonemia associated with urea cycle disorders. This agent may also have a role in the treatment of $\mathrm{HE}$, but clinical trials are needed. ${ }^{51}$

\section{L-Carnitine}

L-Carnitine is shown to stimulate the urea cycle and a delayed onset of HE in hyperammonemic mice. Therrien demonstrated that L-carnitine protects against ammonia neurotoxicity among patients after portacaval-shunt surgery, however, well designed studies are pending. ${ }^{52}$

\section{Molecular adsorbent recirculating system (MARS)}

Molecular adsorbent recirculating system (MARS) is a blood detoxification system based on albumin dialysis. The system removes both protein-bound and water-soluble toxins, which makes it useful for patients with liver failure. The FDA has approved MARS as a toxin removal device in cases of drug overdose and poisoning, however it is not approved for HE. The principal mechanism in MARS therapy is hemodiadsorption, which combines hemodialysis with adsorption using albumin. A prospective, controlled, randomized multicenter trial in patients with HE (grade 3 or 4) was randomized to standard medical therapy or MARS dialysis with standard medical therapy. The MARS dialysis-treated group had a more significant and rapid improvement in their mental status compared to standard medical therapy. ${ }^{53}$

\section{Drug effects on neurotransmission}

\section{Branched-chain amino acids}

Branched-chain amino acids are decreased and aromatic amino acid are increased in patients with cirrhosis 
and HE. This imbalance may competitively inhibit normal neurotransmitters such as dopamine and norepinephrine. ${ }^{54,55}$ Attempts to correct this imbalance with the administration of branched-chain amino acids intravenously and orally have been performed for many decades. In a meta-analysis of five studies using intravenous branched-chain amino acids infusions to treat HE, a significant improvement of HE was observed overall, but in two of the studies there was an increase in mortality. ${ }^{56}$ Large multicenter studies are needed before branched-chain amino acids can be used clinically.

\section{Flumazenil}

Flumazenil, a short-acting benzodiazepine receptor antagonist, has been used as a treatment for patients with $\mathrm{HE}$. There is evidence of an increase in benzodiazepine receptor activation among cirrhotic patients with HE. In a systematic review, Als-Nielsen and colleagues included 12 controlled trials with a total of 765 patients and found flumazenil to be associated with a significant improvement in HE. However, it failed to show any long-term benefits or improvement in survival. ${ }^{57}$ Flumazenil is not routinely used as therapy for HE because of significant side effects including seizures, nausea, vomiting, dizziness, and agitation.

\section{Bromocriptine}

Bromocriptine, which stimulate dopamine receptors, is approved for Parkinson's disease. A reduction in dopaminergic neurotransmission has been proposed as one of the underlying mechanisms for HE. Improvement of extrapyramidal symptoms has been reported when bromocriptine was added to more conventional therapies. ${ }^{58}$ Bromocriptine at $30 \mathrm{mg}$ orally twice daily can be considered in patients who are refractory to other therapies with extrapyramidal symptoms. ${ }^{6}$

\section{Zinc}

Zinc deficiency is common among cirrhotic patients, especially with alcohol-induced liver injury. Zinc supplementation has been shown to decrease serum ammonia levels and altering neurotransmitters like gamma-aminobutyric acid and norepinephrine in the brain. ${ }^{59}$ Only small studies have been performed using zinc supplementation with equivocal results. ${ }^{60,61}$ Zinc is an oral agent that is available over-thecounter and has a role for patients with zinc deficiency. Zinc is available in several forms including zinc gluconate, zinc sulfate, and zinc acetate. The recommended daily allowance of elemental zinc is $11 \mathrm{mg}$ in adult males and $8 \mathrm{mg}$ in adult females. The elemental zinc content appears in the supplemental facts panel on the supplement container. Zinc toxicity can occur in both acute and chronic forms. Acute adverse effects of high zinc intake include nausea, vomiting, loss of appetite, abdominal cramps, diarrhea, and headaches.

\section{Surgical treatments}

Obliteration of large spontaneous portosystemic anastomoses, surgical shunts, or transjugular intrahepatic portosystemic shunt (TIPS) can be a therapeutic option among chronic HE patients. However, the risk of bleeding and hepatic decompensation should be taken into account when those procedures are performed. Splenic artery embolization or total colectomy are other possibilities that have been used in highly selected patients with chronic HE who are resilient to medical therapy. ${ }^{8,11}$

Liver transplantation is the ultimate curative treatment available for liver disease and HE. Appropriate candidates should be referred to a liver transplant center after the first episode of overt encephalopathy due to its poor prognostic sign. ${ }^{4}$ The model for end-stage liver disease (MELD) system used for organ allocation in the United States includes objective data (serum creatinine, total bilirubin, and international normalized ratio of prothrombin time) and does not include any subjective data (including HE). Objective data is used since it verifiable, but excluding subjective data does a disservice to patients with HE and low MELD scores.

\section{Miscellaneous treatments \\ AST- 120}

AST-120 (Kremezin ${ }^{\circledR}$; Kureha Corp., Tokyo, Japan) is an oral adsorbent used in Japan for delaying the initiation of dialysis for patients with chronic kidney disease. ${ }^{62}$ AST-120 adsorbs to toxins and mediators of inflammation from the gastrointestinal tract, including ammonia, bile acids, histamine, serotonin, tyramine, tryptamine and octopamine. It is currently undergoing a phase 2, multicenter, randomized, double-blinded, placebo-controlled, dose-ranging trial to evaluate the efficacy, safety, and tolerability for eight weeks in patients with mild HE. ${ }^{63}$

\section{Probiotics}

Probiotics is a medical therapy that attempts to alter colonic flora for a clinical benefit. Probiotics have been used to treat HE by decreasing urease-producing bacteria and promoting growth of nonurease-producing bacteria. Loguerico and colleagues reported in a pilot study that Enterococcus faecium was as effective as lactulose in reducing ammonia levels and improving mental status among chronic HE patients. ${ }^{64}$ In another pilot study by Liu, probiotics improved cognitive 
functioning in $50 \%$ of patients with minimal HE. ${ }^{65}$ One should note that probiotics are not largely used clinically due to the fear of introducing live bacteria into patients who are in an immunosuppressed state.

\section{Acarbose}

Acarbose is an FDA-approved medication for the treatment of type 2 diabetes mellitus. It inhibits alpha-glucosidase activity in the intestine and delays digestion of ingested carbohydrates. For patients with $\mathrm{HE}$, acarbose can decrease colonic proteolytic flora and the degradation of dietary nitrogenous substances. Gentile and colleagues demonstrated in a randomized, controlled and crossover trial, significant reduction in blood ammonia coupled with clinical improvement of HE in 107 cirrhotic patients treated with acarbose 150-300 mg/day compared to a placebo. ${ }^{66}$ This drug is not routinely used for the management of HE because the product information sheet states that acarbose is contraindicated in patients with cirrhosis.

\section{Manganese}

Manganese deposition in many cirrhotic brains (globus pallidus) has been detected by magnetic resonance imaging (MRI), which disappears after liver transplantation and HE. ${ }^{67}$ Although there are no significant correlations between plasma manganese levels and $\mathrm{HE}$, the similarity in clinical symptoms between $\mathrm{HE}$ and manganese toxicity makes it an interesting area for further studies to evaluate the effects of manganese-chelating agents on HE treatment. ${ }^{68}$

\section{$\mathrm{N}$-methyl-D-aspartate}

$\mathrm{N}$-methyl-D-aspartate (NMDA) receptors can interact with glutamate in the brain to activate many enzymes, including nitric oxide synthetase. Nitric oxide can activate other pathways important to cerebral processes such as circadian rhythms, memory and learning. Hyperammonemia enhances glutamatergic activity and overstimulation of NMDA receptors in the brain. Hyperammonemia induced rats develop neurologic symptoms similar to HE, but many of these symptoms resolve with the administration of NMDA receptor antagonists. ${ }^{69}$ Human studies are still pending.

\section{Conclusions}

The treatment of acute or persistent HE has changed very little during the past forty years. However, with newer agents under investigation, the available treatment options will increase over the next five years. Until then treatment options are limited to correcting or removing precipitating causes, dietary changes and pharmacologic approaches that are focused on the use of nonabsorbable disaccharides. Additional pharmacological agents available include certain antibiotics, drugs to eliminate ammonia from the plasma, and a few other drugs with special indications.

A complete list of precipitating factors associated with acute episodes of $\mathrm{HE}$ or persistent $\mathrm{HE}$ are identified in Table 2. The most common causes include infection, gastrointestinal bleeding, electrolyte abnormalities, dehydration, use of alcohol or benzodiazepines, and constipation. A complete history, physical exam, and laboratory studies should identify most of these causes.

Dietary changes can prevent episodes of acute HE and persistent HE. A diet focused on vegetable-based protein source and high fiber can be associated with developing less HE.

The first line agent used to prevent acute or persistent HE are the nonabsorbable disaccharides (lactulose or lactitol). Clinical trials have established the efficacy of the nonabsorbable disaccharides in enema form to treat acute episodes

Table 2 Common precipitants of hepatic encephalopathy and the underlying mechanisms

I. Increased nitrogen load

a. Excess dietary protein

b. Constipation

c. Gastrointestinal bleeding

d. Blood transfusions

e. Azotemia

f. Infection

g. Hypokalemia

2. Decrease toxins clearance

a. Dehydration

i. Fluid restriction

ii. Excessive diuresis

iii. Abdominal paracentesis

iv. Diarrhea due to laxatives

b. Hypotension

i. Bleeding

ii. Systemic vasodilatation

c. Anemia

d. Porto systemic shunts (iatrogenic and spontaneous)

3. Altered neurotransmission

a. Benzodiazepines

b. Psychoactive drugs

4. Hepatocellular damage

a. Continued alcohol abuse

b. Development of hepatocellular carcinoma 
Table 3 Treatment summary

First line agents: (Level II-I-II-3)

\section{Lactulose or Lactitol}

Acute HE - enemas: $300 \mathrm{ml}$ in $1000 \mathrm{ml}$ every two hours until clinical improvement.

Acute $\mathrm{HE}$ - oral: $45 \mathrm{ml}$ each hour until bowel movement and clinical improvement.

Chronic HE - oral: I5-45 ml tid or bid continuous until two to three bowel movements per day.

Second line agents: (Level I-III)

Rifaximin (Level I)

Chronic HE - oral: 400-550 mg po bid

Neomycin (Level III)

Acute $\mathrm{HE}$ - oral: I g every six hours for up to six days

Chronic HE - oral: I-2 g/day

Metronidazole (Level III)

Chronic HE - oral: $250 \mathrm{mg}$ bid

Third line agents: (Level II-3-III)

Sodium benzoate (Level II-3)

Chronic $\mathrm{HE} \mathrm{-} \mathrm{oral:} \mathrm{titrate} \mathrm{up} \mathrm{to} \mathrm{clinical} \mathrm{improvement} \mathrm{or} \mathrm{a} \mathrm{maximum} \mathrm{dose} \mathrm{of} 5 \mathrm{~g}$ bid

Elemental zinc from zinc gluconate, zinc sulfate, and zinc acetate (Level II-3)

Chronic HE - oral: II mg in adult males and $8 \mathrm{mg}$ in adult females every day

Bromocriptine (Level III)

Chronic HE - oral: 30 mg bid

Fourth line agents: (Level II-3)

Chronic HE - surgical obliteration of large spontaneous portosystemic anatomoses, splenic artery embolization, or total colectomy.

The US Preventive Services Task Force evidence ranking ${ }^{71}$

- Level I: Evidence obtained from at least one properly designed randomized controlled trial.

- Level II- I: Evidence obtained from well-designed controlled trials without randomization.

- Level II-2: Evidence obtained from well-designed cohort or case-control analytic studies, preferably from more than one center or research group.

- Level II-3: Evidence obtained from multiple time series with or without the intervention. Dramatic results in uncontrolled trials might also be regarded as this type of evidence.

- Level III: Opinions of respected authorities, based on clinical experience, descriptive studies, or reports of expert committees.

of HE. Extensive clinical experience has demonstrated the efficacy of oral nonabsorbable disaccharides. Unfortunately, not all patients are able to tolerate these agents. For patients who can not tolerate the sweet taste of lactulose a powder packet is available. For patients who develop gastrointestinal bloating or debilitating diarrhea a second line agent is considered.

The second line agents include oral antibiotics (neomycin, metronidazole, and rifaximin) that can reduce ureaseproducing bacteria in the intestines, resulting in a decrease of ammonia production and absorption through the gastrointestinal tract. Both neomycin and metronidazole have been used for many years to treat $\mathrm{HE}$ and are inexpensive. However, the potential side effects of neomycin (ototoxicity and renal insufficiency) and metronidazole (peripheral neuropathy) limit their use. Rifaximin has minimal side effects and is effective, but the cost of US\$5 for a $200 \mathrm{mg}$ tablet limits its clinical use. ${ }^{70}$

The third line agents available for patients who can not tolerate the above agents or still have persistent or episodic HE on the above agents are sodium benzoate, zinc, or bromocriptine. In addition, patients with unique situations may also benefit from an agent in this class. Sodium benzoate is inexpensive, but is associated with a large sodium load and gastrointestinal side-effects. Most patients can not tolerate the $5 \mathrm{~g}$ twice a day and will need to start at a lower dose and slowly titrate up. The prescription must be filled by a compounding pharmacy and is not always paid for by insurance companies. Zinc is an oral agent that is available over-the-counter and has a role for patients with zinc deficiency. Bromocriptine has been used by the authors with benefit in the patient with extrapyramidal symptoms. 
Patients who are still symptomatic with HE despite all of the above may benefit from surgical options including obliteration of large spontaneous portosystemic anatomoses, splenic artery embolization or total colectomy. HE in cirrhotic patients is associated with a survival lower than $50 \%$ at one year. Therefore liver transplant should be considered in those patients as the ultimate cure, unfortunately the MELD system does not prioritize the allocation of organs to those recipients with HE (Table 3).

\section{Disclosure}

The authors report no conflicts of interest in this work.

\section{References}

1. Mullen K. Hepatic encephalopathy. In: Zakim D, Boyer TD, editors. Hepatology: A Textbook of Liver Disease. 5th Ed. Philadelphia, PA: WB Saunders; 2006. p. 311-331.

2. Riggio O, Efrati C, Catalano C, et al. High prevalence of spontaneous portal-systemic shunts in persistent hepatic encephalopathy. Hepatology. 2005;42:1158-1165.

3. Sanyal AJ, Freedman AM, Shiffman ML, Purdum PP 3rd, Luketic VA, Cheatham AK. Portosystemic encephalopathy after transjugular intrahepatic portosystemic shunt: results of a prospective controlled study. Hepatology. 1994;20(1 Pt 1):46-55.

4. Ferenci P. Hepatic encephalopathy. In: Haubrich WS, Schaffner F, Berk JE, editors. Bockus Gastroenterology. 5th Ed. Philadelphia, PA: WB Saunders; 1995. p. 1998-2003.

5. Bustamante J, Rimola A, Ventura PJ, et al. Prognostic significance of hepatic encephalopathy in patients with cirrhosis. J Hepatol. 1999; 30:890-895.

6. Ferenci P, Lockwood A, Mullen K, et al. Hepatic encephalopathydefinition, nomenclature, diagnosis, and quantification: final report of the working party at the 11 th World Congresses of Gastroenterology, Vienna, 1998. Hepatology. 2002;35:716-721.

7. Blei A, Cordoba J. Practice Parameters Committee of the American College of Gastroenterology: Hepatic encephalopathy. Am J Gastroenterol. 2001;96:1968-1976.

8. Mendler M, Donovan J, Blei A. Central nervous system and pulmonary complications of end-stage liver diseases. In: Yamada J, Alpers D, Kaplowitz N, et al. editors. Textbook of Gastroenterology. 4th Ed. Philadelphia, PA: Lippincott Williams \& Wilkins; 2003. p. 2445-2467.

9. Blei AT, Córdoba J. Hepatic encephalopathy. Am J Gastroenterol. 2001;96:1968-1976.

10. Chatauret N, Butterworth RF. Effects of liver failure on inter-organ trafficking of ammonia: Implications for the treatment of hepatic encephalopathy. J Gastroenterol Hepatol. 2004;19:S219-S223.

11. Ahl B, Weissenborn K, Van den Hoff J, et al. Regional differences in cerebral blood flow and cerebral ammonia metabolism in patients with cirrhosis. Hepatology. 2004;40:73-79.

12. O'Carroll RE, Hayes PC, Ebmeier KP, et al. Regional cerebral blood flow and cognitive function in patients with chronic liver disease. Lancet. 1991;337:1250-1253.

13. Stahl J. Studies of the blood ammonia in liver disease: Its diagnostic, prognostic, and therapeutic significance. Ann Intern Med. 1963; 58:1-24.

14. Blei AT. Hepatic encephalopathy. In: Bircher J, Benhamou JP, McIntyre N, Rizzetto M, Rodés J, editors. Oxford Textbook of Clinical Hepatology. 2nd Ed. Oxford, UK: Oxford Medical; 1999. p. 765-783.

15. Córdoba J, Blei AT. Hepatic encephalopathy. In: Schiff ER, Sorrell MF, Maddrey WC, editors. Schiff's Diseases of the Liver. Philadelphia, PA: Lippincott Williams \& Wilkins; 2003. p. 595-623.
16. Butterworth RF. The neurobiology of hepatic encephalopathy. Semin Liver Dis. 1996;16:235-244.

17. Ahboucha S, Butterworth RF. Pathophysiology of hepatic encephalopathy: A new look at GABA from the molecular standpoint. Metab Brain Dis. 2004;19:331-343.

18. Kircheis G, Wettstein M, Timmermann L, et al. Critical flicker frequency for quantification of low-grade hepatic encephalopathy. Hepatology. 2002;35:357-366.

19. Romero-Gómez M, Córdoba J, Jover R, et al. Value of the critical flicker frequency in patients with minimal hepatic encephalopathy. Hepatology. 2007;45:879-885.

20. Sharma P, Sharma BC, Puri V, Sarin SK. Critical flicker frequency: diagnostic tool for minimal hepatic encephalopathy. $J$ Hepatol. 2007;47:67.

21. Haseler LJ, Sibbitt WL, Mojtahedzadeh HN, et al. Proton MR spectroscopic measurement of neurometabolites in hepatic encephalopathy during oral lactulose therapy. AJNR Am J Neuroradiol. 1998;19: 1681-1686.

22. Blei AT, Cordoba J. Practice Parameters Committee of the American College of Gastroenterology. Hepatic encephalopathy. Am J Gastroenterol. 2001;96:1968-1976.

23. Ferenci P, Müller CH. Hepatic encephalopathy: Treatment. Chapter 26. In: Burroughs A, Feagan B, McDonald JWB, editors. Evidence-Based Gastroenterology. London, UK: British Medical Journal; 1999. p. 443.

24. Weissenborn K, Ennen JC, Schomerus H, et al. Neuropsychological characterization of hepatic encephalopathy. J Hepatol. 2001;34: 768-773.

25. Fessel JN, Conn HO. An analysis of the causes and prevention of hepatic coma. Gastroenterology. 1972;62:191.

26. Córdoba J, López-Hellín J, Planas M, et al. Normal protein diet for episodic hepatic encephalopathy: Results of a randomized study. J Hepatol. 2004;41:38-43.

27. Bianchi GP, Marchesini G, Fabbri A, et al. Vegetable versus animal protein diet in cirrhotic patients with chronic encephalopathy. A randomized cross-over comparison. J Intern Med. 1993;233:385-392.

28. Riordan SM, Williams R. Treatment of hepatic encephalopathy. NEngl J Med. 1997;337:473-479.

29. Gluud C. Branched-chain amino acids for hepaticencephalopathy? Hepatology. 1991;13:812-813.

30. DerSimonian R. Parenteral nutrition with branched chain amino acids in hepatic encephalopathy: Meta analysis. Hepatology. 1990;11: 1083-1084.

31. Als-Nielsen B, Gluud L, Gluud C. Nonabsorbable disaccharides for hepatic encephalopathy. Cochrane Database Syst Rev. 2004;2: CD003044.

32. Prasad S, Dhiman, RK, Duseja A, et al. Lactulose improves cognitive functions and health-related quality of life in patients with cirrhosis who have minimal hepatic encephalopathy. Hepatology. 2007;45: 549-559.

33. Morgan MY, Hawley KE. Lactitol vs lactulose in the treatment of acute hepatic encephalopathy in cirrhotic patients: A double blind, randomized trial. Hepatology. 1987;7:1278-1284.

34. Uribe M, Campollo O, Vargas F. Acidifying enemas (lactitol and lactulose) vs nonacidifying enemas (tap water) to treat acute portalsystemic encephalopathy: A double-blind randomized clinical trial. Hepatology. 1987;7:639-643.

35. Bass N, Mullen K, Sigal S, et al. Rifaximin is effective in maintaining remission in hepatic encephalopathy: Results of a large, randomized, placebo-controlled trial. J Hepatol. 2009;50(Suppl 1):S53.

36. Mas A, Rodés J, Sunyer L, et al; Spanish Association for the Study of the Liver Hepatic Encephalopathy Cooperative Group. Comparison of rifaximin and lactitol in the treatment of acute hepatic encephalopathy: Results of a randomized, double-blind, double-dummy, controlled clinical trial. J Hepatol. 2003;38:51-58.

37. Lawrence KR, Klee JA. Rifaximin for the treatment of hepatic encephalopathy. Pharmacotherapy. 2008;28(8):1019-1032. 
38. Conn HO, Leevy CM, Vlahcevic ZR, et al. Comparison of lactulose and neomycin in the treatment of chronic portal-systemic encephalopathy. Gastroenterology. 1977;72(4 Pt 1):573-583.

39. Strauss E, Tramote R, Silva EP, Caly WR. Double-blind randomized clinical trial comparing neomycin and placebo in the treatment of exogenous hepatic encephalopathy. Hepatogastroenterology. 1992;39:542-545

40. Blei AT, Cordoba J; Practice Parameters Committee of the American College of Gastroenterology. Hepatic encephalopathy. Am J Gastroenterol. 2001;96:1968-1976.

41. Manuel MA, Kurtz I, Saiphoo CS, Nedzelski JM. Nephrotoxicity and ototoxicity following irrigation of wounds with neomycin. Can J Surg. 1979;22(3):274-277.

42. Morgan MH, Read AE, Speller DC. Treatment of hepatic encephalopathy with metronidazole. Gut. 1982;23(1):1-7.

43. Basu P, Rayapudi K, Estevez J, Brown RS. A pilot study utilizing nitazoxanide for hepatic encephalopathy in chronic liver failure [abstract]. San Francisco, CA: Program and abstracts of the 59th Annual Meeting of the American Association for the Study of Liver Diseases; October 31-November 4, 2008. 1742.

44. Mantry PS, Munsaf S. Does the addition of rifaximin to lactulose reduce the severity of hepatic encephalopathy? A single-center experience [abstract]. Hepatology. 2008;48:517A, \#472.

45. Mantry PS, Munsaf S. Comparison of tolerability of adjunctive rifaximin treatment versus lactulose monotherapy for hepatic encephalopathy [abstract]. Hepatology. 2008;48:518A, \#473.

46. Kircheis G, Nilius R, Held C, et al. Therapeutic efficacy of L-ornithine-L-aspartate infusions in patients with cirrhosis and hepatic encephalopathy: Results of a placebo-controlled, double-blind study. Hepatology. 1997;25:1351.

47. Stauch S, Kircheis G, Adler G, et al. Oral L-ornithine L-aspartate therapy of chronic hepatic encephalopathy: Results of a placebocontrolled double-blind study. J Hepatol. 1998;28:856-864.

48. Jalan R, Wright G, Davies NA, Hodges SJ. L-ornithine phenylacetate (OP): a novel treatment for hyperammonemia and hepatic encephalopathy. Med Hypotheses. 2007;69:1064-1069.

49. Mendenhall CL, Rouster S, Marshall L, et al. A new therapy for portal systemic encephalopathy. Am J Gastroenterol. 1986;81:540-543.

50. Sushma S, Dasarathy S, Tandon RK, et al. Sodium benzoate in the treatment of acute hepatic encephalopathy: A double-blind randomized trial. Hepatology. 1992;16:138-144.

51. Batshaw ML, MacArthur RB, Tuchman M. Alternative pathway therapy for urea cycle disorders: twenty years later. J Pediatr. 2001;138 (1 Suppl):S46-S54; discussion S54-S55.

52. Therrien G, Rose C, Butterworth J, Butterworth RF. Protective effect of L-carnitine in ammonia-precipitated encephalopathy in the portacaval shunted rat. Hepatology. 1997;25:551-556.

53. Hassanein TI, Tofteng F, Brown RS Jr, et al. Randomized controlled study of extracorporeal albumin dialysis for hepatic encephalopathy in advanced cirrhosis. Hepatology. 2007;46:1853-1862.

54. Ferenci P, Wewalka F. Plasma amino acids in hepatic encephalopathy. J Neural Transm Suppl. 1978;14(Suppl):87-94.
55. Morgan MY, Milsom JP, Sherlock S. Plasma ratio of valine, leucine and isoleucine to phenylalanine and tyrosine in liver disease. Gut. 1978;19:1068-1073.

56. Naylor CD, O’Rourkee K, Detsky AS, Baker JP. Parenteral nutrition with branched-chain amino acids in hepatic encephalopathy a metaanalysis. Gastroenterology. 1989;97:1033-1042.

57. Als-Nielsen B, Kjaergard LL, Gluud C. Benzodiazepine receptor antagonists for acute and chronic hepatic encephalopathy. Cochrane Database Syst Rev. 2001;4:CD002798.

58. Uribe M, Farca A, Marquez MA, Garcia-Ramos G, Guevara L. Treatment of chronic portal systemic encephalopathy with bromocriptine: A double-blind controlled trial. Gastroenterology. 1979;76:1347-1351.

59. Agus ZS, Dukes ID, Morad M. Divalent cations modulate the transient outward current in rat ventricular myocytes. Am J Physiol. 1991;261: C310-C318.

60. Marchesini G, Fabbri A, Bianchi G, et al. Zinc supplementation and amino acid-nitrogen metabolism in patients with advanced cirrhosis. Hepatology. 1996;23(5):1084-1092.

61. Bresci G, Parisi G, Banti S. Management of hepatic encephalopathy with oral zinc supplementation: a long-term treatment. Eur J Med. 1993;2(7):414-416.

62. Ueda H, Shibahara N, Takagi S, Inoue T, Katsuoka Y. AST-120, an oral adsorbent, delays the initiation of dialysis in patients with chronic kidney diseases. Ther Apher Dial. 2007;11(3):189-195.

63. Hiraishi M, Inoue N, Yamazaki Z, et al. Effects of an oral adsorbent in model of surgically induced liver dysfunction. JPN J Artif Organs. 1987; 16:(2)

64. Loguercio C, Abbiati R, Rinaldi M, et al. Long-term effects of Enterococcus faecium SF68 versus lactulose in the treatment of patients with cirrhosis and grade 1-2 hepatic encephalopathy. $J$ Hepatol. 1995;23(1):39-46.

65. Liu Q, Duan ZP, Ha da K, et al. Synbiotic modulation of gut flora: Effect on minimal hepatic encephalopathy in patients with cirrhosis. Hepatology. 2004;39:1441-1449.

66. Gentile S, Guarino G, Romano M, et al. A randomized controlled trial of acarbose in hepatic encephalopathy. Clin Gastroenterol Hepatol. 2005;3(2):184-191.

67. Solomou E, Velissaris D, Polychronopoulos P, et al. Quantitative evaluation of magnetic resonance imaging abnormalities in subclinical hepatic encephalopathy. Hepatogastroenterology. 2005;52:203-207.

68. Córdoba J, Blei AT. Hepatic encephalopathy. In: Schiff ER, Sorrell MF, Maddrey WC, editors. Schiff's Diseases of the Liver. Philadelphia, PA: Lippincott Williams \& Wilkins; 2003. p. 595-623.

69. Vogels BA, Maas MA, Daalhuisen J, et al. Memantine, a noncompetitive NMDA receptor antagonist improves hyperammonemia-induced encephalopathy and acute hepatic encephalopathy in rats. Hepatology. 1997;25:820-827.

70. Drugstore.com. Online Pharmacy. Pricing data. 2009. Accessed on June 22, 2009. Available from: http://www.drugstore.com/.

71. Agency for Healthcare Research Quality. U.S. Preventive Services Task Force (USPSTF). 2009. Accessed on June 22, 2009. Available from: http://www.ahrq.gov/clinic/uspstfix.htm.
Therapeutics and Clinical Risk Management

\section{Publish your work in this journal}

Therapeutics and Clinical Risk Management is an international, peerreviewed journal of clinical therapeutics and risk management, focusing on concise rapid reporting of clinical studies in all therapeutic areas, outcomes, safety, and programs for the effective, safe, and sustained use of medicines. This journal is indexed on PubMed Central, CAS,
Dovepress

EMBase, Scopus and the Elsevier Bibliographic databases. The manuscript management system is completely online and includes a very quick and fair peer-review system, which is all easy to use. Visit http://www.dovepress.com/testimonials.php to read real quotes from published authors. 